\title{
Subcutaneous Immunoglobulin Use in Inclusion Body Myositis: A Review of 6 Cases
}

\author{
Patrick Cherin ${ }^{a} \quad$ Jean-Christophe Delain ${ }^{c} \quad$ Christophe de Jaeger $^{b}$ \\ Jean-Charles Crave ${ }^{c}$ \\ ${ }^{a}$ Department of Internal Medicine, Pitié-Salpetrière Hospital Group, and ${ }^{b}$ Institut de \\ Médecine et Physiologie de la Longévité, Paris, and ' Octapharma, Boulogne-Billancourt, \\ France
}

\section{Key Words}

Inclusion body myositis - Immunoglobulin - Subcutaneous immunoglobulins · Corticosteroid . Biopsy · Muscle weakness

\begin{abstract}
Introduction: Inclusion body myositis (IBM) is a slowly progressive degenerative inflammatory disorder affecting both proximal and distal muscles. Immunosuppressive therapies are generally ineffective in the treatment of this disorder, and most patients are resistant to steroid therapy. Some benefits with mild improvement were observed with intravenous immunoglobulin (IVIg), particularly in patients with severe dysphagia. Objectives: The objective of this review was to describe the use of subcutaneous Ig (SCIg) in patients with IBM and to assess its feasibility. Results: This report reviews 6 cases of IBM treated with SCIg in clinical practice. All patients had received prior treatments for IBM, including immunosuppressive agents and IVIg. SCIg was administered over a long period of time, ranging from 4.5 to 27 months. No patient discontinued the SCIg because of a treatment-related event or safety issues. The 6 cases showed an improvement in muscle strength and resolution of dysphagia. For 2 patients, this improvement persisted for approximately 12 months. Conclusions: SCIg might be proposed as an alternative therapy to patients with IBM who are resistant to corticoids and immunosuppressive therapies. Our findings suggest that treatment with SCIg (Gammanorm 16.5\%, Octapharma AB) is feasible and safe in patients with IBM.
\end{abstract}


Cherin et al.: Subcutaneous Immunoglobulin Use in Inclusion Body Myositis: A Review of 6 Cases

\section{Introduction}

Inclusion body myositis (IBM) is a complex disorder of unknown etiology, where inflammation and autoimmunity against muscle fibers coexists with features of degeneration [1]. IBM is a slowly progressive degenerative inflammatory disorder affecting both proximal and distal muscles, resulting in substantial weakness and atrophy. It may affect axial muscles, resulting in camptocormia or head-drop. At late-stage disease, pharyngeal muscles could be affected, leading to life-threatening dysphagia and aspiration pneumonia and finally death. Histologically, IBM is characterized by the presence of endomysial CD8 inflammatory cells surrounding myofibers and rimmed vacuoles, and an abnormally elevated number of cytochrome oxidase-negative fibers [2].

Some patients might initially respond to steroid therapy; however, the large majority remains resistant [3]. Similarly, immunosuppressive therapies, including methotrexate, cyclosporine, azathioprine or mycophenolate mofetil, are largely ineffective in patients with IBM [4]. Therefore, the efficacy of intravenous immunoglobulin (IVIg) was evaluated in patients with IBM [5-8]. Some benefits with mild improvement were observed in IVIg-treated patients; however, improvement was unsustainable and no changes in muscle histology were noticed. Particularly, few studies suggested some benefit in patients with severe dysphagia $[7,9,10]$.

Subcutaneous Ig (SCIg) is increasingly used as an alternative to IVIg. It is indicated for primary (and some secondary) immune deficiencies and is recommended in some neurological autoimmune diseases such as peripheral neuropathies and myositis. This paper reviews 6 cases of IBM treated with SCIg (Gammanorm 16.5\%, Octapharma AB, Stockholm, Sweden).

\section{Case 1}

A 63-year-old woman presented with a 4-year history of myalgia related to statin therapy. Myalgia persisted more than 1 year after statin discontinuation in 2010. Muscle testing showed mild proximal weakness, and laboratory studies found elevated serum creatine kinase $(1,400 \mathrm{IU} / \mathrm{l}$, normal values $<170 \mathrm{IU} / \mathrm{l})$. An electromyography (EMG) identified a myogenic pattern, and magnetic resonance imaging (MRI) revealed inflammation disseminated in the gluteal and adductor muscles. A muscle biopsy did not show any particular signs, and capillaroscopy results were normal.

In January 2011, a diagnosis of polymyositis was made. Initially, a treatment with methotrexate (15 mg daily) and corticoids (30 mg daily) was started, and then the dose was progressively reduced. A transient clinical and biological improvement was observed. Recurrence of myalgia was reported in May 2011, with an increase in creatine kinase levels in August 2011. These circumstances led to the introduction of the IVIg treatment. The patient received a 6-month therapy of IVIg at $60 \mathrm{~g}$ monthly. A partial decrease in pain without significant clinical improvement was noticed.

In November 2011, the patient was referred to our clinic where a diagnosis of IBM was confirmed by muscle biopsy. The patient was able to move almost normally; however, precise movements were difficult to perform without help (i.e. to open a bottle). She had a poor appetite and had lost 4-5 kg during the last months. She was exercising daily to increase muscle strength, despite an increased asthenia and difficulties in climbing and descending stairs, and she had some problems in swallowing. She reported a diffuse arthralgia affecting particularly the joints in the hands and knees. The EMG confirmed a persistent myogenic pattern. MRI demonstrated an extensive high short-tau inversion recovery signal abnormali- 
Cherin et al.: Subcutaneous Immunoglobulin Use in Inclusion Body Myositis: A Review of 6 Cases

ty in the musculature of the adductor and fasci with fat replacement and amyotrophia. The muscle strength score was $65 / 88$ (88 points corresponds to normal muscle power) and the creatine phosphokinase (CPK) level was elevated (216 IU/l).

Upon the diagnosis of IBM, the patient was initiated on SCIg $(2 \mathrm{~g} / \mathrm{kg} / \mathrm{month})$ in March 2012. She received 18 infusions during 4.5 months ( 2 infusions/week). CPK levels remained above the standard levels (378 IU/l after 4 months of treatment). She reported mild headaches during the SCIg treatment, but the treatment was generally well tolerated. Despite an improvement in muscle strength (muscle strength score from 65 to 70/88), the treatment with SCIg was discontinued because of reimbursement refusal, and IVIg was thus reintroduced.

\section{Case 2}

A 74-year-old woman presented with IBM diagnosed in 2003. She had a history of hypertension and chronic posttransfusion hepatitis $C$ discovered in 2002. Corticosteroid therapy was contraindicated; therefore, she received IVIg as a first-line therapy $(120 \mathrm{~g} / \mathrm{month}$ over 2 days).

In 2010, the patient presented with chronic proximal leg and distal arm asymmetric muscle weakness. EGM did not record any motor activity in the vastus lateralis; the highest activity was recorded in the right vastus medialis. MRI showed a persistent bilateral atrophy of the quadriceps, with rare inflammatory hypersignal. Physical therapy (twice weekly) was thus introduced. Despite a good tolerance of IVIg, the patient requested to shift to SCIg. She had normal CPK levels (158 IU/l).

In August 2011, she was initiated on SCIg administered at $1.2 \mathrm{~g} / \mathrm{kg} / \mathrm{month}$ (2 infusions/week and $120 \mathrm{ml} /$ week). In October 2012, the posology was reduced to $40 \mathrm{ml} /$ week. During the treatment, the patient complained of swelling and pain at the injection site. The patient was stable for almost 12 months, and then a significant decrease in muscle power was observed at 18 months (from 64 to $56 / 88$ for the muscle power score). The patient was barely able to walk. The muscle disability scale (MDS) was stable over 12 months: $27 / 75$ at SCIg initiation and 26/75 after 12 months ( 75 points being the maximum disability). The patient received 115 SCIg infusions over a total period of 27 months.

\section{Case 3}

A 71-year-old woman was diagnosed with polymyositis in March 2005. The patient had a history of hypertension, two breast cancers and a thyroid nodule.

At disease onset, symptoms included a progressive weakness of the lower extremities, perceived particularly after physical activity. A biopsy performed in the quadriceps muscle led to the diagnosis of polymyositis. Corticosteroid therapy (progressively tapered to $5 \mathrm{mg} /$ day) and immunosuppressive therapy with mycophenolate mofetil (CellCept 1,500 $\mathrm{mg} /$ day) was introduced in 2007. However, these treatments were not particularly efficient. The patient progressed, presenting a distal motor deficit and low back pain, which contributed to postural instability and resulted in several falls. The MRI showed significant inferior quadriceps atrophy and hypersignal T2 in 2008. IVIg therapy $(2 \mathrm{~g} / \mathrm{kg} / \mathrm{month})$ was initiated in 2008 with moderate benefits and was stopped in 2010.

The disease was slowly progressing; a slight dysphagia was reported in 2010. The biopsy performed in April 2011 showed inflammatory exudates surrounding and invading 
Cherin et al.: Subcutaneous Immunoglobulin Use in Inclusion Body Myositis: A Review of 6 Cases

nonnecrotic muscle fibers accompanied by rimmed vacuoles, characteristic of IBM. Immunosuppressive therapy (CellCept) was discontinued. She presented with weakness and walking difficulty and reported dyspnea on exertion. CPK was increasing as well (271 IU/l). SCIg was thus initiated on November 25, 2011, as IVIg was only slightly beneficial. SCIg was given at $2 \mathrm{~g} / \mathrm{kg} / \mathrm{month}$ ( 2 infusions/week and $190 \mathrm{ml} /$ infusion). She received 43 infusions within 11 months. The disease was stable during the 5 first months with complete resolution of dysphagia. The muscle power score was between 70 and 74/88, and CPK was between 271 and 336 IU/l. In September 2012, she showed a general clinical deterioration including walking difficulties and recurrence of swallowing troubles. She stopped SCIg in November 2012 due to surgical aneurysm repair. She did not report any adverse event during the SCIg therapy.

\section{Case 4}

A 69-year-old man with a history of hypertension initially complained of bilateral symmetrical muscle weakness in the arms which began in 2011 and then extended to the legs, resulting in walking difficulty. A diagnosis of lumbar spinal stenosis was made, and the patient underwent a laminectomy in January 2013. However, the symptoms exacerbated, and worsening muscle weakness was observed. A muscle biopsy and MRI were performed, and the results confirmed the diagnosis of IBM. Corticosteroid therapy was thus initiated at 40 $\mathrm{mg} /$ day, in association with physical therapy, and was decreased later to $20 \mathrm{mg} / \mathrm{day}$.

Despite the corticosteroid therapy, CPK levels were increased and the patient reported difficulties climbing stairs or rising from a seated position. The muscle power score was 66/88 and the MDS score was 14/75. In October 2013, SCIg was initiated (3 infusions/week, $2 \mathrm{~g} / \mathrm{kg} / \mathrm{month}$ ) and corticosteroids were maintained (5 mg/day). In January 2014, he reported a significant clinical improvement; the muscle power score was at 81/88 and the MDS at 6/75. The patient received 62 infusions over a period of 15 months. After almost 2 years, an increase in body weight was observed (1.5 kg), and the disease was stable (muscle power score 78 and MDS 8). No adverse events were reported.

\section{Case 5}

A 52-year-old HIV-positive man presented with a 2-year history of fatigue and difficulties climbing stairs. A muscle MRI performed in 2013 showed the presence of myositis, and EMG displayed a myogenic pattern. A muscle biopsy performed in January 2013 demonstrated characteristic patterns of IBM. Biological tests showed elevated CPK $(2,943 \mathrm{IU} / \mathrm{l})$. The patient showed symptom worsening; muscle pain associated with a proximal and less distal deficit and swallowing difficulty were reported. The muscle power score was 77/88. A decision to initiate SCIg was made in agreement with the nephrology division. The patient was initiated on SCIg in March 2014 at $2 \mathrm{~g} / \mathrm{kg} / \mathrm{month}$ (2 perfusions/week, $180 \mathrm{ml} /$ perfusion). The patient received 24 infusions over a period of 6 months. The treatment was well tolerated, and the patient showed a mild clinical improvement (muscle power score 75/88 and MDS 13/75 at 6 months); dysphagia had resolved. 
Cherin et al.: Subcutaneous Immunoglobulin Use in Inclusion Body Myositis: A Review of 6 Cases

\section{Case 6}

A 70-year-old man presented with a diagnosis of polymyositis made in 2011 following a 3-year history of progressive muscle weakness. A muscle biopsy, performed at the initial diagnosis, showed inflammatory infiltrates surrounding necrotic muscle fibers and abundant cytochrome oxidase-negative fibers, but without rimmed vacuoles. Corticosteroid therapy was thus initiated at $1 \mathrm{mg} / \mathrm{kg} /$ day and was progressively reduced to $20 \mathrm{mg}$ daily. An improvement was reported, particularly for swallowing troubles. An MRI performed in September 2011 showed muscle atrophy, moderate fatty infiltration and a slight hypersignal. In December 2011, the patient started a physical therapy program, which resulted in a general clinical improvement and reduced pain.

In January 2013, the patient reported a general deficit worsening, with dysphagia, fatigue and generalized pain. Despite corticosteroid therapy, a muscle biopsy showed an active myositis with a T2 hypersignal, increased atrophy and fatty degeneration.

In February 2014, another biopsy confirmed the IBM diagnosis, and SCIg was shortly initiated at $2 \mathrm{~g} / \mathrm{kg} / \mathrm{month}$ ( 2 perfusions/week, $200 \mathrm{ml} /$ perfusion). At the latest visit, he had been treated over almost 21 months and had received 86 infusions. The patient experienced skin reactions and headache, but these events did not result in treatment discontinuation. SCIg was well tolerated, and clinical improvement was observed, although he still presented some gait problems. Dysphagia had resolved. The muscle power score increased from 61 to $71 / 88$ and the MDS showed mild disability (12/75). CPK levels were slightly decreased (from 330 to $214 \mathrm{IU} / \mathrm{l}$ ).

\section{Discussion}

This study reviews 6 cases diagnosed with IBM and treated with SCIg. The aim of this review was to describe the use of SCIg in clinical practice in patients with IBM and to assess its feasibility. All patients had received prior treatments for IBM, including steroids, immunosuppressive agents and IVIg. SCIg was mainly initiated in these patients due to swallowing disorders, as reported elsewhere [7, 9, 10]. SCIg was administered over a long period of time, ranging from 4.5 to 27 months. The patient (case 4) who received 18 infusions of SCIg (4.5 months) discontinued the treatment because of reimbursement refusal. No patient discontinued SCIg treatment because of a treatment-related event or safety issues. These results suggest that treatment with SCIg is feasible and safe in patients with IBM.

Few studies have been performed on IBM, with a limited number of patients. Previous data showed that IBM is a rare, disabling disorder with slow progression, leading potentially to wheelchair use and then death due to aspiration pneumonia and complications due to patients being bedridden [11, 12]. Immunosuppressant therapies were not shown to be of benefit, and their risk/benefit ratio should be established [4, 13, 14]. Few studies assessing IVIg therapy suggested some benefit particularly in reducing and stabilizing severe dysphagia $[7,9,10,15]$. Our experience suggests that SCIg could be beneficial in patients with IBM; the 6 cases showed an improvement in muscle strength and resolution of dysphagia. For 2 patients, this improvement persisted for approximately 12 months before clinical exacerbation. In IVIg treatment, one single large dose is given every 3-4 weeks, whereas in SCIg treatment smaller doses once or twice a week are administered. Therefore, fractionating the total dose into smaller portions decreases the changes in serum IgG levels, maintaining sustained serum IgG levels, which offer a better therapeutic effect in patients with IBM, and reducing systemic side effects of IVIg. 
Cherin et al.: Subcutaneous Immunoglobulin Use in Inclusion Body Myositis: A Review of 6 Cases

No serious adverse events were reported. Few mild adverse events were experienced by the patients; however, these events did not lead to treatment discontinuation.

In conclusion, this report showed that SCIg treatment is feasible and safe in patients with IBM. Treatment was generally well tolerated and was given over a long period. Its use might be beneficial in this population. Other studies with a larger number of patients are needed to confirm these findings. However, due to the limited therapeutic choices in IBM, SCIg appears to be an alternative treatment.

\section{Disclosure Statement}

The authors declare that there are no conflicts of interest.

\section{References}

1 Dalakas MC: Sporadic inclusion body myositis - diagnosis, pathogenesis and therapeutic strategies. Nat Clin Pract Neurol 2006;2:437-447.

2 Chahin N, Engel AG: Correlation of muscle biopsy, clinical course, and outcome in PM and sporadic IBM. Neurology 2008;70:418-424.

-3 Needham M, Mastaglia FL: Sporadic inclusion body myositis: a continuing puzzle. Neuromuscul Disord 2008;18:6-16.

4 Mowzoon N, Sussman A, Bradley WG: Mycophenolate (CellCept) treatment of myasthenia gravis, chronic inflammatory polyneuropathy and inclusion body myositis. J Neurol Sci 2001;185:119-122.

5 Dalakas MC: Controlled studies with high-dose intravenous immunoglobulin in the treatment of dermatomyositis, inclusion body myositis, and polymyositis. Neurology 1998;51:S37-S45.

6 Dalakas MC: Mechanism of action of intravenous immunoglobulin and therapeutic considerations in the treatment of autoimmune neurologic diseases. Neurology 1998;51:S2-S8.

7 Dalakas MC, Koffman B, Fujii M, Spector S, Sivakumar K, Cupler E: A controlled study of intravenous immunoglobulin combined with prednisone in the treatment of IBM. Neurology 2001;56:323-327.

-8 Walter MC, Lochmuller H, Toepfer M, Schlotter B, Reilich P, Schroder M, Muller-Felber W, Pongratz D: Highdose immunoglobulin therapy in sporadic inclusion body myositis: a double-blind, placebo-controlled study. J Neurol 2000;247:22-28.

-9 Cherin P, Pelletier S, Teixeira A, Laforet P, Simon A, Herson S, Eymard B: Intravenous immunoglobulin for dysphagia of inclusion body myositis. Neurology 2002;58:326.

10 Pars K, Garde N, Skripuletz T, Pul R, Dengler R, Stangel M: Subcutaneous immunoglobulin treatment of inclusion-body myositis stabilizes dysphagia. Muscle Nerve 2013;48:838-839.

11 Cox FM, Titulaer MJ, Sont JK, Wintzen AR, Verschuuren JJ, Badrising UA: A 12-year follow-up in sporadic inclusion body myositis: an end stage with major disabilities. Brain 2011;134:3167-3175.

$\$ 12$ Lindberg C, Oldfors A: Prognosis and prognostic factors in sporadic inclusion body myositis. Acta Neurol Scand 2012;125:353-358.

13 Mastaglia FL, Phillips BA, Zilko P: Treatment of inflammatory myopathies. Muscle Nerve 1997;20:651-664.

14 Benveniste O, Guiguet M, Freebody J, Dubourg O, Squier W, Maisonobe T, Stojkovic T, Leite MI, Allenbach Y, Herson S, et al: Long-term observational study of sporadic inclusion body myositis. Brain 2011;134:31763184 .

15 Dobloug C, Walle-Hansen R, Gran JT, Molberg 0: Long-term follow-up of sporadic inclusion body myositis treated with intravenous immunoglobulin: a retrospective study of 16 patients. Clin Exp Rheumatol 2012;30:838-842. 